\title{
A randomized trial to evaluate the impact of copra meal hydrolysate on gastrointestinal symptoms and gut microbiome
}

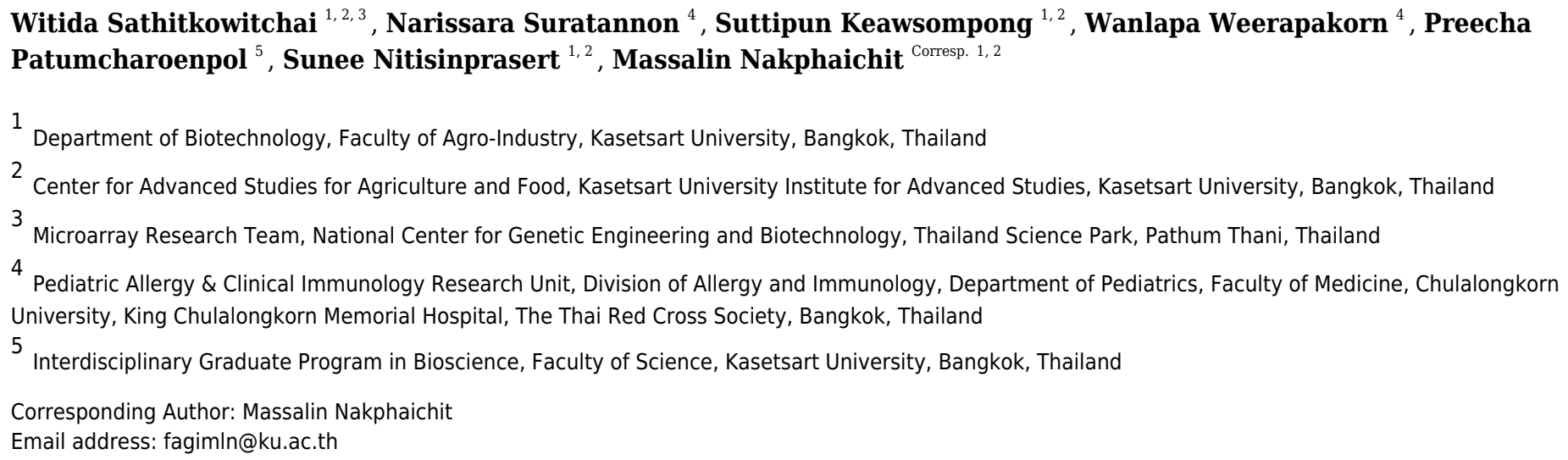

The impact of copra meal hydrolysate $(\mathrm{CMH})$ on gut health was assessed by conducting a double-blind, placebo-controlled study. Sixty healthy adult participants aged 18-40 (BMI = $21-22 \mathrm{~kg} / \mathrm{m}^{2}$ ) were assigned $3 \mathrm{~g} \mathrm{CMH}, 5 \mathrm{~g} \mathrm{CMH}$ or a placebo of drink powder to consume daily for 21 days. Consumption of $\mathrm{CMH}$ at $3 \mathrm{~g} / \mathrm{d}$ improved defecating conditions by reducing stool size and also relieved flatulence and bloating symptoms. Fecal samples were collected serially at the baseline before treatment, after the treatment period and after a 2-week washout period. The gut microbiomes were remarkably similar across the treatment groups, with, microbial community changes observed within the groups. Intake of $\mathrm{CMH}$ at $3 \mathrm{~g} / \mathrm{d}$ led to increased microbial diversity and richness. Reduction was observed in the ratio between Firmicutes to Bacteroidetes, that related to reduced risk of obesity. The $3 \mathrm{~g} / \mathrm{d}$ CMH treatment increased beneficial microbes in the group of fiber-degrading bacteria, especially human colonic Bacteroidetes. While induction of Bifidobacteriaceae was observed after the washout period. Intake of $\mathrm{CMH}$ led to increased lactic acid production, while $3 \mathrm{~g} / \mathrm{d}$ supplementation promoted Immunoglobulin A ( $\lg A)$ in stool samples. A $3 \mathrm{~g}$ daily dose of $\mathrm{CMH}$ led to a potentially beneficial effect on gut health for healthy individuals. 
1

2

3 Witida Sathitkowitchai ${ }^{\mathrm{a}, \mathrm{b}, \mathrm{c}}$, Narissara Suratannon ${ }^{\mathrm{d}}$, Suttipun Keawsompong, ${ }^{\mathrm{a}, \mathrm{b}}$, Wanlapa

4 Weerapakorn $^{\mathrm{d}}$, Preecha Patumcharoenpol ${ }^{\mathrm{e}}$, Sunee Nitisinprasert ${ }^{\mathrm{a}, \mathrm{b}}$, Massalin Nakpaichit ${ }^{\mathrm{a}, \mathrm{b} *}$

5

6 7 Thailand

$8{ }^{b}$ Center for Advanced Studies for Agriculture and Food, Kasetsart University Institute for

9 Advanced Studies, Kasetsart University, Bangkok, Thailand

\section{A randomized trial to evaluate the impact of copra meal hydrolysate on gastrointestinal} symptoms and gut microbiome

aDepartment of Biotechnology, Faculty of Agro-Industry, Kasetsart University, Bangkok

'Microarray Research Team, National Center for Genetic Engineering and Biotechnology, Thailand Science Park, Pathum Thani, Thailand

dPediatric Allergy \& Clinical Immunology Research Unit, Division of Allergy and Immunology, Department of Pediatrics, Faculty of Medicine, Chulalongkorn University, King Chulalongkorn Memorial Hospital, The Thai Red Cross Society, Bangkok, Thailand

eInterdisciplinary Graduate Program in Bioscience, Faculty of Science, Kasetsart University, Bangkok, Thailand.

*Corresponding author, Department of Biotechnology, Faculty of Agro-Industry, Kasetsart University, Bangkok 10900, Thailand.

E-mail address: fagimln@ku.ac.th (M. Nakphaichit). 


\section{Abstract}

The impact of copra meal hydrolysate $(\mathrm{CMH})$ on gut health was assessed by conducting a double-blinded, placebo-controlled study. Sixty healthy adult participants, aged 18-40 years were assigned to daily consume $3 \mathrm{~g}$ of $\mathrm{CMH}, 5 \mathrm{~g}$ of $\mathrm{CMH}$ or placebo in the form of drink powder for 21 days. Consumption of $\mathrm{CMH}$ at $3 \mathrm{~g} / \mathrm{d}$ improved defecating conditions by reducing stool size and also relieved flatulence and bloating symptoms. Fecal samples were collected serially at the baseline before treatment, after the treatment and after a 2-week washout period. The gut microbiomes were similar among the treatment groups, with microbial community changes observed within the groups. Intake of $\mathrm{CMH}$ at $3 \mathrm{~g} / \mathrm{d}$ led to increase microbial diversity and richness. Reduction of the ratio between Firmicutes to Bacteroidetes was observed, although it was not significantly different between the groups. The $3 \mathrm{~g} / \mathrm{d} \mathrm{CMH}$ treatment increased beneficial microbes in the group of fiber-degrading bacteria, especially human colonic Bacteroidetes, while induction of Bifidobacteriaceae was observed after the washout period. Intake of $\mathrm{CMH}$ led to increase lactic acid production, while $3 \mathrm{~g} / \mathrm{d}$ supplement promoted the present of immunoglobulin A (IgA) in stool samples. The $3 \mathrm{~g}$ daily dose of $\mathrm{CMH}$ led to the potentially beneficial effects on gut health for healthy individuals.

Keywords: Copra meal hydrolysate (CMH), prebiotic, mannooligosaccharides, human gut microbiome, short-chain fatty acid 


\section{Introduction}

The human intestine contains trillions of microbes that have been linked to many aspects of health and disease, including metabolic syndrome, infectious diseases and noncommunicable diseases (Jarett et al. 2019). Prebiotics are considered to be functional foods that can modulate the gut microbiome (Prayoonthien et al. 2019). The definition of prebiotics by The International Scientific Association of Probiotics and Prebiotics (ISAPP) is "a substrate that is selectively utilized by host microorganisms conferring a health benefit" (Gibson et al. 2017). Prebiotics increase levels of beneficial bacteria and positively impact the composition of resident microbiota, while also promoting microbe-derived metabolites or signal molecules such as short chain fatty acids (SCFAs) that are important for gut health (Gibson et al. 2017; MarkowiakKopeć \& Śliżewska 2020; Topping 1996). Prebiotics have a mild laxative effect on bowel habits due to indigestible properties. Some prebiotics, especially inulin and oligofructose, alleviate constipation by increasing the fecal bulk (Andersson et al. 2001; Cummings \& Macfarlane 2002).

Copra meal contains galactomannan as a byproduct from the coconut milk industry. To improve the functional food properties of copra meal, galactomannan was successfully hydrolyzed to mannooligosaccharides (MOS) by the enzyme $\beta$-mannanase (Pangsri et al. 2015; Rungruangsaphakun \& Keawsompong 2018; Titapoka et al. 2007). Copra meal hydrolysate $(\mathrm{CMH})$ has been hypothesized as a potential prebiotic. $\mathrm{CMH}$ was stable under upper human gastrointestinal tract conditions (Prayoonthien et al. 2019). In vitro human fecal fermentation showed that $\mathrm{CMH}$ promoted the growth of Lactobacilli and Bifidobacteria similar to fructooligosaccharides (FOS). Induction of two beneficial microbes enhanced SCFAs production 
65 (Prayoonthien et al. 2019). CMH also suppressed pathogenic bacteria such as Salmonella,

Escherichia coli, Staphylococcus aureus and Shigella dysenteriae (Prayoonthien et al. 2019).

Comparing with other prebiotics, documentation of the health-promoting effect of MOS on humans is limited. Impact of MOS from coffee hydrolysate revealed that MOS supplementation over for three weeks promoted the growth of Lactobacilli and Bifidobacteria (Walton et al. 2010). MOS derived from coffee mannan reduced fat absorption in humans (Asano et al. 2006), while MOS reduced the concentration of triglycerides in serum, and subsequently inhibited absorption of lipids in subjects fed a high-fat diet (Kumao et al. 2006).

No official dose recommendation of prebiotics in human diet has been reported. Most prebiotics require an oral dose of $3-5 \mathrm{~g} / \mathrm{d}$ to confer a gut health benefit, depending on prebiotic structure. Daily dosages are suggested at around $5 \mathrm{~g}$ for fructooligosaccharides (FOS), galactooligosaccharides (GOS) and plant sources of prebiotics (Gibson et al. 2017). This is the first clinical study to evaluate the impact of daily consumption of $\mathrm{CMH}$ over three weeks on the gut microbiome, defecating conditions, and gastrointestinal symptoms in healthy volunteers.

Different doses of daily consumption were also investigated.

\section{Materials and Methods}

\section{Preparation of copra meal hydrolysate and placebo drinks}

Copra meal hydrolysate $(\mathrm{CMH})$ was prepared following Rungruangsaphakun \& Keawsompong (2018). The $\mathrm{CMH}$ powder contained 9.7\% crude protein, $1 \%$ crude fat, $14.5 \%$ crude fiber, $12.3 \%$ ash and $53 \%$ nitrogen-free extract. The nitrogen-free extract was composed of 3-6 molecules of MOS, mainly mannose, linking together. Amount of total oligosaccharide of $\mathrm{CMH}$ was $14.77 \%$; mainly mannooligosaccharide with 3-6 degrees of polymerization. The CMH 
87 drinks were prepared in three formulas: the placebo group (maltodextrin $10 \mathrm{~g}$ ), the $3 \mathrm{~g} \mathrm{CMH}$ group

88 ( $3 \mathrm{~g} \mathrm{CMH}$ and $7 \mathrm{~g}$ maltodextrin) and the $5 \mathrm{~g} \mathrm{CMH}$ group ( $5 \mathrm{~g} \mathrm{CMH}$ and $5 \mathrm{~g}$ maltodextrin). The

89 products were packaged in individual daily-use sachets of identical appearance and completely

90 dissolved in room temperature drinking water before consumption.

\section{Study design}

92

This study was a randomized double-blinded placebo-controlled trial with a three-arm parallel group design in 1:1:1 ratio as the placebo group, $\mathrm{CMH} 3 \mathrm{~g}$ (3CMH group) and $\mathrm{CMH} 5 \mathrm{~g}(5 \mathrm{CMH}$ group) (Figure 1). Healthy adults, aged between 18 and 40 years with a body mass index (BMI) of 18.5 to $25.0 \mathrm{~kg} / \mathrm{m}^{2}$, were enrolled (Table S1). This study excluded those who (1) received any antibiotics or anti-viral agents within 3 months before the study, (2) received probiotics/prebiotics within 1 month before the study, and (3) were allergic to coconut and soybean which were components of the study product. Recruitment was conducted through poster and web-based advertising. The study was carried out at the outpatient clinic of King Chulalongkorn Memorial Hospital, Bangkok, Thailand. Physicians and study nurse who involved in the study explained the protocol and enrolled participants to the study. Written informed consent was obtained from all participants. The study was approved by the Ethics Committee of King Chulalongkorn Memorial Hospital, Bangkok, Thailand, under approval reference number 388/61 and registered on the Thai Clinical Trials Registry (TCTR20190426003).

\section{Sample size calculation}

The sample size was determined using the tests for two independent means (two-tailed test) as formula described below. On the basis of previously published study of the effect of MOS from coffee mannan on fecal microflora (Asano et al. 2006), the mean change of stool Bifidobacterium from baseline of subjects received MOS $3 \mathrm{~g}(\mu 1)$ was $20 \%$, while for those received MOS $1 \mathrm{~g}(\mu 2)$ was $10 \%$. The difference $(\Delta)$ was used to calculate, with a power of approximately $80 \%$ (beta 0.2 ) and a 
110 significance level of alpha 0.05 . The ratio (r) was 1 . SD was estimated to be 8 . The numbers of study

111 participants to be included was 11 subjects per group. With the anticipation of 20\% drop out rate, at

112 least 14 subjects per group should be included. So, in our study, we included 20 subjects per group.

$$
\begin{aligned}
& n_{1}=\frac{\left(z_{1-\frac{\alpha}{2}}+z_{1-\beta}\right)^{2}\left[\sigma_{1}^{2}+\frac{\sigma_{2}^{2}}{r}\right]}{\Delta^{2}} \\
& r=\frac{n_{2}}{n_{1}}, \Delta=\mu_{1}-\mu_{2}
\end{aligned}
$$

\section{Randomization and allocation concealment}

Each eligible participant was randomized to 1 of 3 groups and assigned a unique study number.

A blocked randomization list was created using Sealed Envelope Ltd.

117 (https://www.sealedenvelope.com/simple-randomizer/v1/lists), with block sizes of 6 by the statistician

118 who was not involved in the study. Study nurse who did not aware of the code group assigned

119 participants to interventions.

120

121

122

123

124

125

126

127

\section{Intervention}

After randomization, participants were subjected to a 2-week baseline period, 3-week treatment period and 2-week washout period with instruction to use the products once daily during the treatment period (Figure 2). Participants were asked to fill out a daily stool record chart and a 24-hour diary record 3 times per week ( 2 weekdays and 1 day during weekend) throughout the study period. Dietary restrictions were imposed throughout the entire study period (total 49 days), with prohibition of consumption of other fermented products, probiotics, prebiotics and synbiotics supplements to limit potential interference with evaluation of the testing product. The participants were excluded from the study if (1) received dietary supplements described above during the study, (2) received any antibiotics or anti-viral agents during the study and (3) failed to collect stool samples according to the study protocol. Each participant visited the clinic weekly throughout the study. Stool samples were collected 
131 at the baseline visit, the end of treatment, and washout periods. Subjects and researchers who had

132 access to the outcomes of the study were blinded to the code group assigned to participants.

\section{Stool record chart}

134 The stool record charts, adapted from Walton et al. (2010), were recorded daily by all 135 participants throughout the study, in order to investigate whether the products affected the stool 136 frequency, diarrhea, constipation, and gastrointestinal discomfort (for examples bloating symptoms) 137 of the subjects (Table S2). Each stool characteristic was scored as a number and calculated as amean $138( \pm \mathrm{SD})$ per week.

\section{24-hour dietary record}

The 24-hour dietary records were filled in by the subjects 3 times per week ( 2 weekdays and 1 day during weekend) throughout the study period. Energy and nutritive values were calculated using the nutrient calculation computer software INMUCAL-Nutrients V4.0 database NB. 4 (Food Composition Database for INMUCAL Program, 2018) (Table S3).

\section{Stool collection and processing} washout period for analysis of microbiota, SCFAs and secretory immunoglobulin A (IgA). A stool 147 sample of $20 \mathrm{~g}$ was collected in a $76 \times 20 \mathrm{~mm}$ sterile container and immediately placed on ice for transfer to storage at $-80^{\circ} \mathrm{C}$. Stool samples were prepared following the method of Kisuse et al. (2018) for microbiota and SCFA analyses. The fecal samples were diluted 10-fold with phosphate-buffered saline ( $\mathrm{pH}$ 8.0) using a stomacher blender (Stomacher ${ }^{\circledR} 80$ Biomaster, Seward, Worthing, UK) for 5 min. A $1 \mathrm{ml}$ sample of fecal slurry was then centrifuged at $13,000 \times g$ for $5 \mathrm{~min}$., and the supernatant 
152 was stored at $-80^{\circ} \mathrm{C}$ for subsequent SCFA analysis. For microbial DNA extraction, $1 \mathrm{ml}$ of fecal slurry

153 was placed in a $1.5 \mathrm{ml}$ centrifuge tube and then stored at $-80^{\circ} \mathrm{C}$.

\section{Microbial DNA extraction and 16S rRNA gene sequencing}

155 Microbial DNA was extracted according to the modified method of Mirsepasi et al. (2014), 156 utilizing a bead meter and a QIAamp® DNA stool mini kit (Qiagen GmbH, Germany). Qualification 157 and quantification of DNA were determined using a Nanodrop Spectrophotometer (Thermo Fisher 158 Scientific, USA). Extracted DNA samples were immediately stored at $-20^{\circ} \mathrm{C}$.

The variable region of $\mathrm{V} 3-\mathrm{V} 4$ of $16 \mathrm{~S}$ rRNA genes was amplified using the forward primer

160

161

162

163

164

165

166

167

168

169

170

171

172

173

174

Imina-V3-V4-F (5'-TCGTCGGCAGCGTCAGATGTGTATAAGAGACAGCCTACGGGNGGC WGCAG-3') and reverse primer Imina-V3-V4-R (5'- GTCTCGTGGGCTCGGAGATGTGTAT AAGAGACAGGACTACTACHVGGGTATCTAATCC-3'). Cycling conditions consisted of an initial denaturation at $94^{\circ} \mathrm{C}$ for $2 \mathrm{~min}$, followed by 25 cycles of denaturation at $94{ }^{\circ} \mathrm{C}$ for 20 seconds, annealing at $57{ }^{\circ} \mathrm{C}$ for 30 seconds, extension at $72{ }^{\circ} \mathrm{C}$ for 30 seconds, and a final extension at $72{ }^{\circ} \mathrm{C}$ for 10 min. The amplified products were purified using NucleoSpin ${ }^{\circledR}$ Gel and PCR Clean-up (Macherey-Nagel Inc., USA) according to the manufacturer's protocol and sent to the Omics Sciences and Bioinformatics Center (Faculty of Science, Chulalongkorn University, Bangkok, Thailand) for further sequencing using the Illumina MiSeq platform (USA).

\section{Processing of 16S rRNA gene sequences and data analysis}

The raw sequences were processed using bioinformatics tools. Paired-end reads were first quality trimmed using BBDUK (read quality $>15$ at 3'), and the primer at the 5' end was removed using seqtk (https://github.com/lh3/seqtk). Any obtained sequence shorter than 150 bp was excluded along with its pair. Filtered pairs of sequences were corrected, merged and chimeras were removed using the DADA2 pipeline (v1.10) (Callahan et al. 2016). High-quality sequences were 
175 obtained at $41,843 \pm 10,553$ reads per sample. Taxonomy was identified using the QIIME2

176 classifier (v2019.1) (Bolyen et al. 2019) with the Greengenes version 13.8 database. Cutoff

177 confidence was indicated at 0.7 .

178

179

180

181

182

183

184

185

186

187

188

189

190

191

192

193

194

195

The PICRUSt2 pipeline package (v2.1.0_b) was used to predict the functional potential of gut microbiomes from observable 16S rRNA sequences. The KEGG pathway levels were inferred according to the instructions from the PICRUSt2 website (Douglas \& Maffei 2020).

\section{Alpha and beta diversity analysis}

Alpha diversity (Chao1, Shannon) and beta diversity were calculated using the Vegan package of R software (version 2.5.6). Statistical differences in the diversity indices among the three groups were identified using the Kruskal-Wallis H test. Beta-diversity among participants was visualized via non-metric multidimensional scaling (NMDS) ordination based on Bray-Curtis dissimilarity matrices.

\section{Short-chain fatty acid analysis}

Lactic acid, acetic acid, butyric acid, and propionic acid from the fecal slurries were analyzed using high-performance liquid chromatography (HPLC) (Water 1525, USA). Samples were prepared following the modified method of Wang et al. (2019). The supernatant was collected using centrifugation at $13,000 \times \mathrm{g}$ at $4{ }^{\circ} \mathrm{C}$ for $5 \mathrm{~min}$ and mixed with $0.2 \% \mathrm{v} / \mathrm{v}$ tartaric acid as the internal standard (ratio 3:1). Before injection, the mixed solution was filtered through a $0.2 \mu \mathrm{m}$ PVDF filter (Verical, Thailand). SCFAs were isolated using an Aminex HPX-87H column (300 x 7.8 mm BioRad, USA) at $50{ }^{\circ} \mathrm{C}$, with $8 \mathrm{mM}$ sulfuric acid used as the mobile phase at a flow rate of $0.60 \mathrm{ml} / \mathrm{min}$ for $60 \mathrm{~min}$ and wavelength $210 \mathrm{~nm}$ using a UV detector (Waters 2489, USA). The injector was set with a split ratio of 20:1. Agilent Technologies 7890A equipment (Santa Clara, USA) was used for 
196 data collection and calculation of all parameters. Standard solutions were prepared from lactic acid,

197 acetic acid, propionic acid and butyric acid at concentrations of 1, 2, 2.5, 5, 10 and $20 \mathrm{mmol} / \mathrm{ml}$.

198 Stool secretory IgA

199

200

201

202

203

204

205

206

207

208

209

210

211

212

213

214

215

216

217

Analysis of secretory immunoglobulin A (IgA) in stool samples was performed in 55 out of the 56 subjects who completed the study (19 in $3 \mathrm{CMH}, 17$ in $5 \mathrm{CMH}, 19$ in placebo group). Samples were collected at the baseline and after the treatment period and stored at $-80{ }^{\circ} \mathrm{C}$. All fecal samples were thawed at room temperature and weighed to $0.1 \mathrm{~g}$. Phosphate-buffered saline was added to the samples, then they were mixed in a stomacher machine and centrifuged at $14,000 \mathrm{rpm}$ at $4{ }^{\circ} \mathrm{C}$ for $15 \mathrm{~min}$. A sample of $200 \mu \mathrm{l}(10 \mathrm{X})$ of supernatant was collected and diluted to $200 \mathrm{X}$ for the single plex human isotyping assay. IgA was measured using a Bio-Plex Pro $^{\text {TM }}$ Human Isotyping kit (cat no. 171A3101M, Bio-Rad, USA). The Bio-Plex Pro ${ }^{\mathrm{TM}}$ assays were essentially immunoassays formatted on magnetic beads by captured antibodies directed against the desired biomarker coupled to the beads. The coupled beads reacted with samples containing the IgA biomarker of interest, and biotinylated detection antibody was added to create a sandwich complex. The final detection complex was formed with addition of streptavidinphycoerythrin conjugated using the Bio-Plex ${ }^{\circledR} 200$ system reader (Bio-Rad, USA). The concentration of analyte bound to each bead was proportional to the median fluorescence intensity of the reporter signal.

\section{Statistical analysis}

Statistical analyses were performed using the statistical software package SPSS version 17. Statistical significance among the three groups was determined using one way ANOVA and the Kruskal-Wallis test for parametric and non-parametric tests, respectively. Comparisons of the two 
218 groups were determined using the independent t-test and Mann-Whitney U-test for parametric and

219 non-parametric tests, respectively.

220 Linear mixed models of bacterial relative abundance were performed for each participant

221 using R package (2.15.0) including age, gender, carbohydrate intake, fiber intake, dose of CMH

222 supplement and sampling period.

\section{Results}

\section{Demographic characteristics of study subjects}

Of the 227 subjects screened, 60 were enrolled in the project at visit 1 and 56 completed

the study (Figure 1). The drop out occurred randomly due to the use of antibiotics and anti-viral

treatment of subjects, which happened to occur in $\mathrm{CMH}$ group. Another drop-out subject was

due to the non-compliance of stool collection. Therefore, the higher drop out in CMH groups

was unlikely related to the testing products. The participants were randomly assigned to the three subgroups, each of which had similar demographic characteristics (Table 1). Participants in the

$3 \mathrm{CMH}$ group were older than those in the placebo group. During the testing period, energy, and

nutrient consumption values of the placebo, $3 \mathrm{CMH}$ and $5 \mathrm{CMH}$ groups were calculated (Table 2).

Results showed that all subjects consumed similar carbohydrate, protein, fat, fiber, and sugar contents. Therefore, the energy consumption was also similar in all groups.

\section{Influence of CMH on defecating conditions and gastrointestinal symptoms}

Defecating conditions and gastrointestinal symptoms in the baseline period of all groups

were similar (Table S4). Interestingly, stool size of participants in the 3CMH group was 
240 from the $5 \mathrm{CMH}$ group was also smaller than that from the placebo group but did not reach

241 statistical significance. Participants in the 3CMH and 5CMH groups showed improvement in

242 defecating conditions including less strong smell, softened stools and reduced gastrointestinal

243 discomfort (such as bloating, flatulence and abdominal discomfort) compared to the placebo

244 group during the testing and washout period but without statistical significance (Table S4).

245 Median changes of defecating and gastrointestinal symptoms between the baseline and

246 testing period or washout period were analyzed. Two factors including size of stool and

247 flatulence/bloating symptoms showed significant difference between sample groups (Figure 3).

248 Comparing with the baseline, size of stool and flatulence/bloating symptoms of the 3CMH group

249 reduced and reduction level was significantly lower than the placebo group $(p=0.059)$.

250 Similarly, reduction of stool size and flatulence/bloating symptoms in the 3CMH group was

251 observed in the washout period when compared to the baseline. Median level change was also

252 significantly lower than the placebo group $(p=0.045)$. Unlike the 3CMH group, median changes

253 of stool size and flatulence/bloating symptoms from baseline to testing period in the $5 \mathrm{CMH}$

254 group were similar to the placebo group. Flatulence and bloating symptoms were significantly

255 greater in the 5CMH group at washout period compared with the baseline and significantly lower

256 than the placebo group $(p \leq 0.05)$.

\section{Influence of CMH on microbial richness and diversity}

Microbial richness indices of stool samples in each group were monitored during the

baseline, testing and washout periods (Figure S1). The Chao1 index of the $3 \mathrm{CMH}$ group during

260 the testing and washout periods increased compared to the baseline, while the Chao indices for

261 the placebo and 5CMH groups decreased and were stable, respectively. 
during the testing and washout periods compared to the baseline (Figure S1), while Shannon

264

265

266

267

268

269

270

271

272

273

274

275

276

277

diversity indices of the $5 \mathrm{CMH}$ and placebo groups during the baseline, testing and washout

periods were stable. These results suggested that the administration of $\mathrm{CMH}$ at $3 \mathrm{~g} / \mathrm{d}$ promoted microbial diversity and richness.

The microbial communities from all groups and time points were intermingled in nonmetric multidimensional scaling plots using Bray-Curtis dissimilarity (Figure S1). Similar to the result of alpha diversity, beta-diversity showed no differences or clusters due to $\mathrm{CMH}$ intake or sampling period.

\section{Influence of CMH on fecal microbiota}

To assess specific changes in gut microbiota caused by $\mathrm{CMH}$, gut microbiota from different taxa were identified from the three sampling periods (baseline, testing, and washout) (Table S5, S6 and S7). Six microbiota phyla (Actinobacteria, Bacteroides, Firmicutes, Lentisphaerae, Proteobacteria and Verrucomicrobia) were found in all groups (Table S8). The most dominant phyla in each period were Firmicutes, Bacteroides and Actinobacteria. Dynamic changing patterns during the baseline, testing and washout periods of all phyla from the three groups were similar. No significant changes were recorded in the microbial phyla in the $\mathrm{CMH}$ treatment. It was observed that Firmicutes and Bacteroidetes ratios at the testing period for both the $3 \mathrm{CMH}$ and $5 \mathrm{CMH}$ groups were lower than the placebo, although they were not significantly different (Table S8).

At the family level, changes in gut microbiota during testing period were similar in all groups (Figure 4A). Supplementation with 3CMH promoted various microbes including

Desulfovibrionaceae, Bifidobacteriaceae, Barnesiellaceae, Veillonellaceae, Lachnospiraceae,

Peer] reviewing PDF | (2021:02:58215:2:0:NEW 21 Aug 2021) 
285

286

287

288

289

290

291

292

293

294

295

296

297

298

299

300

301

302

303

304

305

306

307

Clostridiaceae, Rikenellaceae, Prevotellaceae, Porphyromonadaceae, Paraprevotellaceae,

Odoribacteraceae, and Bacteroidaceae, while eight families of gut microbiota in the placebo and $5 \mathrm{CMH}$ groups were stable (mean change $=0$ ) and six families decreased. Ruminococcaceae was reduced in the $3 \mathrm{CMH}$ group, whereas it increased in the placebo and 5CMH groups. Median change in Ruminococcaceae in the placebo group was significantly greater than in the $3 \mathrm{CMH}$ group $(p \leq 0.05)$.

After the washout period, median changes in gut microbiota were similar in all groups (Figure 4B). However, induction of Bifidobacteriaceae was observed in the 3CMH group even after the products were discontinued, while showing reduction in the placebo and 5CMH groups. The amount of Enterobacteriaceae reduced in the $3 \mathrm{CMH}$ and placebo groups, with significantly different median change compared with the 5CMH group $(p \leq 0.05)$, that showed an increase during the washout period.

To confirm the relationship of changes of three families including Ruminococcaceae, Bifidobacteriaceae and Enterobacteriaceae with $\mathrm{CMH}$ intake, the linear mixed effect model was performed (Table S9). Results revealed that gender, age, sampling period, carbohydrate and fiber consumption had no significant effect on changes in the three families. Supplementation with 3CMH showed a significant effect on Ruminococcaceae observed during testing period, while with 5CMH significantly affected Bifidobacteriaceae and Enterobacteriaceae during the washout period $(\mathrm{p} \leq 0.05)$

At the testing period, predictions of functional analysis of gut microbiota were focused on carbohydrate-related metabolism (Figure 5). Among the fifteen pathways, median change in relative abundance of the two pathways, ascorbate/aldarate and fructose/mannose metabolism showed significant differences between the three groups. The gene function associated with 
308

309

310

311

312

313

314

315

316

317

318

319

320

321

322

323

324

325

326

327

328

ascorbate/aldarate and fructose/mannose metabolism in the 3CMH group significantly increased

more than in the placebo group $(p \leq 0.05)$. However, median changes in the $5 \mathrm{CMH}$ group were

similar to those in the placebo group, indicating that $\mathrm{CMH}$ intake enhanced the microbial

pathways of ascorbate/aldarate and fructose/mannose metabolism.

\section{Influence of CMH on fecal short-chain fatty acids}

The major fecal metabolite in all samples were acetic acid, followed by butyric acid, propionic acid, and lactic acid (Table 3). The SCFAs in all subjects at the baseline were similar. Intake of $\mathrm{CMH}$ had no effect on the four SCFAs and they were similar during the testing period; however, significant differences among the three groups were observed during the washout period. Highest abundance of lactic acid was recorded in the $5 \mathrm{CMH}$ group followed by the $3 \mathrm{CMH}$ and placebo groups $(\mathrm{p}<0.05)$. The amount of lactic acid in the $5 \mathrm{CMH}$ group was significantly higher than in the placebo group but similar to the $3 \mathrm{CMH}$ group. The level of butyric acid in the placebo group was significantly greater than in the $3 \mathrm{CMH}$ group.

\section{Influence of secretory immunoglobulin A in stools}

Results revealed no significant differences in secretory IgA levels in stools among all groups both at the baseline and after the testing period (Table 4). Forty-two percent of the participants who received $3 \mathrm{CMH}$ treatment showed increased stool secretory IgA compared to $20 \%$ of the participants in the $5 \mathrm{CMH}$ and placebo groups. The increase in IgA correlated with increased stool frequency $(r=0.303$ to 0.039$)$ and reduced abdominal discomfort symptoms $(r=$ 0.238 ) during the treatment period.

\section{Discussion}


The health effects of dietary fibers have been accepted worldwide (Carlson et. al. 2018).

330 However, health effects of CMH have not been well defined, especially in human. To address

331

332

333

334

335

336

337

338

339

340

341

342

343

344

345

346

347

348

349

350

351 this lack of knowledge, a randomized, double-blinded placebo-controlled design was used to determine the impact on gut health of $\mathrm{CMH}$ containing mannooligosaccharides. Two different dosages of $\mathrm{CMH}, 3$ and $5 \mathrm{~g} / \mathrm{d}$, were investigated for gastrointestinal symptoms and defecating condition. Consumption of $\mathrm{CMH} 3 \mathrm{~g} / \mathrm{d}$ improved defecating condition and gastrointestinal symptoms by reducing the size of stools and ameliorating flatulate/bloating symptoms (Table S4). A smaller stool is easier to expel and improves discomfort (Bannister et al. 1987). This effect was still observed after discontinuing $\mathrm{CMH}$ consumption. Prebiotics at a dose of $\leq 6 \mathrm{~g} / \mathrm{d}$ reduced flatulence but a higher dose might cause intestinal bloating, pain, flatulence, or diarrhea (Gibson et al. 2010; Moretti et al. 2018; Piemontese et al. 2011; Scholtens et al. 2014; Wilson et al. 2019). In our study, intake of 5 grams of CMH per day did not induce any flatulence/bloating or discomfort, while a dose of $3 \mathrm{~g} / \mathrm{d}$ had more impact on gastrointestinal symptoms.

Prebiotics contribute to change in the gut microbial community by acting as a primary carbon source. Several reports revealed that prebiotics enhanced the intestinal microbial community (Carlson et. al. 2018). Our results suggested that consumption of CMH at both 3 and $5 \mathrm{~g} / \mathrm{d}$ did not significantly change the gut microbial richness and diversity (Figure S1), which tended to increase after the testing period in the $3 \mathrm{CMH}$ group, with higher levels still maintained after the washout period.

Only a few microbiome phyla changed as the result of $\mathrm{CMH}$ treatments (Table S8). The Firmicutes/Bacteroidetes ratio of the $3 \mathrm{CMH}$ and $5 \mathrm{CMH}$ groups after the testing period tended to be lower than in the placebo group. A high Firmicutes/Bacteroidetes ratio has been linked with overweight and obesity (Castaner et al. 2018; Verdam et al. 2013). A positive effect of MOS on

Peer] reviewing PDF | (2021:02:58215:2:0:NEW 21 Aug 2021) 
352 gut microbiota modulation of obesity and fat metabolism has been reported. Intake of MOS, in

353 mice fed with a high-fat diet, decreased the Firmicutes/Bacteroidetes ratio (Wang et al. 2018).

354 Moreover, coffee MOS also inhibited the intestinal absorption of dietary fat and enhanced fat

355 excretion in healthy adults (Kumao \& Fujii 2006; Kumao et al. 2005).

357 Enterococcus spp. was observed in CMH gut model fermentation (Prayoonthien et al. 2018;

358 Prayoonthien et al. 2019). In a human trial, intake of $\mathrm{CMH}$ at $3 \mathrm{~g} / \mathrm{d}$ enhanced human colonic

359 Bacteroidetes including Rikenellaceae, Prevotellaceae, Porphyromonadaceae,

360 Paraprevotellaceae, Odoribacteraceae, and Bacteroidaceae. These bacteria contained

361 hemicellulose-degrading enzymes that hydrolyzed xylans, mannans and galactomannans (Flint et

362 al. 2008; Flint et al. 2012; Holscher et al. 2015). Intake of $3 \mathrm{~g} / \mathrm{d}$ of CMH promoted several

363 intestinal microbes than the 5CMH group except for Ruminococcaceae.

fructose/mannose metabolism in the $3 \mathrm{CMH}$ group increased after the testing period, with significantly higher median change than in the placebo group. Change in fructose/mannose metabolism in the $5 \mathrm{CMH}$ group also increased after the testing period but was lower than in the 368 3CMH group. Induction of Bifidobacteriaceae was not observed after the testing period (Figure 4), but was found in the $3 \mathrm{CMH}$ group during the washout period. This result suggested that $\mathrm{CMH}$ treatment indirectly introduced the conditions for enrichment of Bifidobacteriaceae. This trend was more evident in the washout period. Induction of Bifidobacteriaceae during the washout 
374 explanation for this is selective utilization in small doses but loss of activity when in excess, as

375 other microbes may become involved in fermentation (Walton et al. 2010).

Prebiotics are selectively fermented by the gut microbiome to produce SCFAs but effects

of CMH on SCFA production remain unclear in human studies (Table 3). SCFA measurement in

feces was not reflected the amount within the colon and $95 \%$ of SCFAs produced by the gut microbiome were absorbed (Walton et al. 2010). However, levels of lactic acid in feces of the $3 \mathrm{CMH}$ group were higher than in the placebo. microbiome (Hemarajata \& Versalovic 2013). The daily consumption of $3 \mathrm{~g}$ of CMH promoted the increase level of IgA, whereas no induction effect was observed following a high dose of $\mathrm{CMH}$. Moreover, the percentage of subjects whose stool IgA value increased was higher in the $3 \mathrm{CMH}$ group than in the placebo and $5 \mathrm{CMH}$ groups. This result was consistent with other studies reporting that daily consumption of MOS at $1-3 \mathrm{~g}$ was more effective on the gut microbiome and immune modification than a higher dosage (Asano et al. 2004; Kumao et al. 2006; Umemura et al. 2004a; Umemura et al. 2004b; Walton et al. 2010). limitations. The lack of significant improvement in gut health could be attributed to the small sample size of the intervention study. Participants in the placebo group were mostly younger than the treatment groups. Our research samples were mostly from healthy adult females (77\%). Future clinical studies are needed to provide on gender and age associated disparities in the 394 effect of $\mathrm{CMH}$ on defecating conditions, gastrointestinal symptoms, and gut microbiome 395 modulation. 
396

397

398

399

400

401

402

403

404

405

406

407

408

409

410

411

412

413

414

415

416

417

\section{Conclusion}

$\mathrm{CMH}$ is a novel potential source of prebiotics and has shown health benefits in human studies. Consumption of $\mathrm{CMH}$ at $3 \mathrm{~g} / \mathrm{d}$ improved defecating conditions and gastrointestinal symptoms. Intake of $\mathrm{CMH}$ at $3 \mathrm{~g} / \mathrm{d}$ also improved the gut microbiome by increasing beneficial microbes, their metabolites and host immunity. Our results suggest that $\mathrm{CMH}$ has a prebiotic effect when taken at $3 \mathrm{~g} / \mathrm{d}$ by healthy individuals. This finding can also increase $\mathrm{CMH}$ value for functional food industry application.

\section{Acknowledgement}

The authors thank Assoc. Prof. Dr. Wanwipa Vongsangnak and Assoc. Prof. Dr. Sunee

Nitisinprasert for valuable comments and discussion. The authors also acknowledge the Division of Allergy and Immunology, Department of Pediatrics, Faculty of Medicine, Chulalongkorn University for laboratory facilities and cohort study.

\section{Reference}

Andersson H, Asp N-G, Bruce Å, Roos S, Wadström T, and Wold A. 2001. Health effects of probiotics and prebiotics A literature review on human studies. Food \& Nutrition Research 45. 10.3402/fnr.v45i0.1790

Asano I, Fujii S, Kaneko M, Takehara I, and Fukuhara I. 2006. Investigation of mannooligosaccharides blended coffee beverage on abdominal fat reduction in humans. Japanese Journal of Medical and Pharmocologic Science 55:93-103.

Asano I, Umemura M, Fujii S, Hoshino H, and Iino H. 2004. Effects of Mannooligosaccharides from Coffee Mannan on Fecal Microflora and Defecation in Healthy Volunteers. Food 
420

421

Bannister JJ, Davison P, Timms JM, Gibbons C, and Read NW. 1987. Effect of stool size and consistency on defecation. Gut 28:1246-1250. 10.1136/gut.28.10.124

Bolyen, E., Rideout, J. R., Dillon, M. R., Bokulich, N. A., Abnet, C. C., Al-Ghalith, G. A., ... and Caporaso, J. G. 2019. Reproducible, interactive, scalable and extensible microbiome data science using QIIME 2. Nature biotechnology, 37(8), 852-857.6

Callahan, B. J., McMurdie, P. J., Rosen, M. J., Han, A. W., Johnson, A. J. A. and Holmes, S. P. 2016. DADA2: high-resolution sample inference from Illumina amplicon data. Nature methods, 13(7), 581-583.Castaner O, Goday A, Park YM, and Lee SH. 2018. The Gut Microbiome Profile in Obesity: A Systematic Review. 2018:4095789. $10.1155 / 2018 / 4095789$

Carlson, J. L., Erickson, J. M., Lloyd, B. B. and Slavin J. L. 2018. Health effect and sources of prebiotic dietary fiber. Curr Dev Nutr. 10.1093/cdn/nzy005

Cummings J, and Macfarlane G. 2002. Gastrointestinal effects of prebiotics. The British journal of nutrition 87 Suppl 2:S145-151. 10.1079/BJNBJN/2002530

Douglas GM, and Maffei VJ. 2020. PICRUSt2 for prediction of metagenome functions. 38:685688. $10.1038 / \mathrm{s} 41587-020-0548-6$

Flint HJ, Bayer EA, Rincon MT, Lamed R, and White BA. 2008. Polysaccharide utilization by gut bacteria: potential for new insights from genomic analysis. Nature Reviews Microbiology 6:121-131. 10.1038/nrmicro1817

Flint HJ, Scott KP, Duncan SH, Louis P, and Forano E. 2012. Microbial degradation of complex carbohydrates in the gut. Gut Microbes 3:289-306. 10.4161/gmic.19897 
441 Gibson GR, Hutkins R, Sanders ME, Prescott SL, Reimer RA, Salminen SJ, Scott K, Stanton C,

442

443

444

445

446

447

448

449

450

451

452

453

454

455

456

457

458

459

460

461

Swanson KS, Cani PD, Verbeke K and Reid G. 2017. Dietary prebiotics: Expert

consensus document: The International Scientific Association for Probiotics and

Prebiotics (ISAPP) consensus statement on the definition and scope of prebiotics. Nat Rev Gastroenterol Hepatol 14:491-502.

Hemarajata P, and Versalovic J. 2013. Effects of probiotics on gut microbiota: mechanisms of intestinal immunomodulation and neuromodulation. Therap Adv Gastroenterol 6:39-51. $10.1177 / 1756283 \times 12459294$

Holscher HD, Caporaso JG, Hooda S, Brulc JM, Fahey GC, Jr., and Swanson KS. 2015. Fiber supplementation influences phylogenetic structure and functional capacity of the human intestinal microbiome: follow-up of a randomized controlled trial. Am J Clin Nutr 101:55-64. 10.3945/ajcn.114.092064

Jarett J, Carlson A, Serao M, Strickland J, Serfilippi L, and Ganz H. 2019. Diets with and without edible cricket support a similar level of diversity in the gut microbiome of dogs. PeerJ 7:e7661. 10.7717/peerj.7661

Kisuse J, La-ongkham O, Nakphaichit M, Therdtatha P, Momoda1 R, Tanaka M, Fukuda S, Popluechai S, Kespechara K, Sonomoto K, Lee Y.K, Nitisinprasert S, and Nakayama J. 2018. Urban diets linked to gut microbiome and metabolome alterations in children: A comparative cross-sectional study in Thailand. Front. Microbiol. 9:1-16.

Kumao T, and Fujii S. 2006. Mannooligosaccharides Blended Coffee Beverage Intake Increases the Fat Level in Feces. J HEALTH SCI 52:329-332. 10.1248/jhs.52.329

Peer] reviewing PDF | (2021:02:58215:2:0:NEW 21 Aug 2021) 
462 Kumao T, Fujii S, Asakawa A, Takehara I, and Fukuhara I. 2006. Effect of Coffee Drink

463 Containing Mannooligosaccharides on Total Amount of Excreted Fat in Healthy Adults.

464 Journal of Health Science - J HEALTH SCI 52:482-485. 10.1248/jhs.52.482

465 Kumao T, Fujii S, Ozaki K, and Takao I. 2005. Effects of diets containing

466

467

468

469

470

471

472

473

474

475

476

477

478

479

480

481

482

483

484 mannooligosaccharides from coffee mannan on fat in blood serum and liver fat in (ZUC)fa/fa rats. Jap J Med Pharm Sci 54:505-509.

La-ongkham O, Nakphaichit M, Nakayama J, Keawsompong S and Nitisinprasert S. 2020. Agerelated changes in the gut microbiota and the core gut microbiome of healthy Thai humans. 3Biotech. 10:276.

Markowiak-Kopeć P, and Śliżewska K. 2020. The Effect of Probiotics on the Production of Short-Chain Fatty Acids by Human Intestinal Microbiome. Nutrients 12. 10.3390/nu12041107

Mirsepasi H, Persson S, Struve C, Andersen LO, Petersen AM, and Krogfelt KA. 2014. Microbial diversity in fecal samples depends on DNA extraction method: easyMag DNA extraction compared to QIAamp DNA stool mini kit extraction. BMC Res Notes 7:50. $10.1186 / 1756-0500-7-50$

Moretti E, Rakza T, Mestdagh B, Labreuche J, and Turck D. 2018. The bowel movement characteristics of exclusively breastfed and exclusively formula fed infants differ during the first three months of life. Acta Paediatrica 108. 10.1111/apa.14620

Pangsri P, Piwpankaew Y, Ingkakul A, Nitisinprasert S, and Keawsompong S. 2015. Characterization of mannanase from Bacillus circulans NT 6.7 and its application in mannooligosaccharides preparation as prebiotic. Springerplus 4:771. 10.1186/s40064015-1565-7

Peer] reviewing PDF | (2021:02:58215:2:0:NEW 21 Aug 2021) 
485 Piemontese P, Giannì ML, Braegger CP, Chirico G, Grüber C, Riedler J, Arslanoglu S, van 486 Stuijvenberg M, Boehm G, Jelinek J, and Roggero P. 2011. Tolerance and safety

487

488

489

490

491

492

493

494

495

496

497

498

499

500

501

502

503

504

505

506

507 evaluation in a large cohort of healthy infants fed an innovative prebiotic formula: a randomized controlled trial. PLoS One 6:e28010. 10.1371/journal.pone.0028010

Prayoonthien P, Nitisinprasert S, and Keawsompong S. 2018. In vitro fermentation of copra meal hydrolysate by chicken microbiota. 3 Biotech 8:41. 10.1007/s13205-017-1058-1

Prayoonthien P, Rastall RA, Kolida S, Nitisinprasert S, and Keawsompong S. 2019. In vitro fermentation of copra meal hydrolysate by human fecal microbiota. 3 Biotech 9:93. $10.1007 / \mathrm{s} 13205-019-1633-8$

Rungruangsaphakun J, and Keawsompong S. 2018. Optimization of hydrolysis conditions for the mannooligosaccharides copra meal hydrolysate production. 3 Biotech 8:169. $10.1007 / \mathrm{s} 13205-018-1178-2$

Scholtens PA, Goossens DA, and Staiano A. 2014. Stool characteristics of infants receiving short-chain galacto-oligosaccharides and long-chain fructo-oligosaccharides: a review. World J Gastroenterol 20:13446-13452. 10.3748/wjg.v20.i37.13446

Titapoka S, Keawsompong S, Haltrich D, and Nitisinprasert S. 2007. Selection and characterization of mannanase-producing bacteria useful for the formation of prebiotic manno-oligosaccharides from copra meal. World Journal of Microbiology and Biotechnology 24:1425-1433. 10.1007/s11274-007-9627-9

Topping DL. 1996. Short-chain fatty acids produced by intestinal bacteria. Asia Pac J Clin Nutr $5: 15-19$.

Umemura M, Fujii S, Asano I, Hoshino H, and Iino H. 2004a. Effect of "Coffee Mix Drink" Containing Mannooligosaccharides from Coffee Mannan on Defecation and Fecal 

TECHNOL RES 10:195-198. 10.3136/fstr.10.195

510 Umemura M, Fujii S, Asano I, Hoshino H, and Iino H. 2004b. Effect of Small Dose of

511 Mannooligosaccharides from Coffee Mannan on Defecating Conditions and Fecal Microflora. Food Science and Technology Research - FOOD SCI TECHNOL RES 10:174-179. 10.3136/fstr.10.174

514 Verdam F, Fuentes S, de Jonge C, Zoetendal E, Erbil R, Greve JW, Buurman W, De Vos W, and Rensen S. 2013. Human intestinal microbiota composition is associated with local and systemic inflammation in obesity. Obesity (Silver Spring, Md) 21. 10.1002/oby.20466

Walton G, Rastall RA, Martini MC, Williams CE, Jeffries RL, and Gibson GR. 2010. A doubleblind, placebo controlled human study investigating the effects of coffee derived mannooligosaccharides on the faecal microbiota of a healthy adult population. International Journal of Probiotics and Prebiotics 5:75-83.

Wang H, Zhang X, Wang S, Li H, Lu Z, Shi J, and Xu Z. 2018. Mannan-oligosaccharide modulates the obesity and gut microbiota in high-fat diet-fed mice. Food Funct 9:39163929. $10.1039 / \mathrm{c} 8$ fo00209f

Wang HY, Wang C, Guo LX, Zheng YF, Hu WH, Dong TTX, Wang TJ, and Tsim KWK. 2019.

Wilson B, Rossi M, Dimidi E, and Whelan K. 2019. Prebiotics in irritable bowel syndrome and other functional bowel disorders in adults: a systematic review and meta-analysis of randomized controlled trials. Am J Clin Nutr 109:1098-1111. 10.1093/ajcn/nqy376 


\section{Table 1 (on next page)}

Demographic characteristics of study participants at baseline

All values are expressed as mean \pm SD. ${ }^{a, b}$ Significance between sample groups by the Kruskal-Wallis test are indicated by different lowercase superscripts ( $p \leq 0.05)$. 
1 Table 1 Demographic characteristics of study participants at baseline.

\begin{tabular}{lcccc}
\hline Variable & Placebo & 3CMH & 5CMH & p value \\
\hline Number & 20 & 19 & 17 & 0.998 \\
Age (year) & $27.7 \pm 4^{\mathrm{b}}$ & $32 \pm 5^{\mathrm{a}}$ & $31 \pm 5^{\mathrm{ab}}$ & 0.041 \\
Sex (female/male) & $15 / 5$ & $15 / 4$ & $13 / 4$ & 0.958 \\
Body mass index $\left(\mathrm{kg} / \mathrm{m}^{2}\right)$ & $21.6 \pm 2.1$ & $22 \pm 2$ & $21 \pm 2$ & 0.231 \\
\hline
\end{tabular}

2

3 All values are expressed as mean $\pm \mathrm{SD}$

4 a,bSignificance between sample groups by the Kruskal-Wallis test are indicated by different

5 lowercase superscripts $(p \leq 0.05)$.

6 


\section{Table 2 (on next page)}

Energy and nutrient consumption of placebo, $3 \mathrm{CMH}$ and $5 \mathrm{CMH}$ subjects during testing period.

All values are expressed as median and interquartile range (IQR) 
1 Table 2 Energy and nutrient consumption of placebo, $3 \mathrm{CMH}$ and $5 \mathrm{CMH}$ subjects during the 2 testing period.

\begin{tabular}{lcccc}
\hline \multicolumn{3}{c}{ Item } & \multicolumn{3}{c}{ Average intake/day } & \multirow{p}{p}{} \\
& Placebo $(\mathbf{n}=\mathbf{2 0})$ & $\mathbf{3 C M H ~ ( n = 1 9 )}$ & $\mathbf{5 C M H ~ ( n = 1 7 )}$ & value \\
\cline { 2 - 4 } & $153.95(135.98,236.54)$ & $160.89(163.54,206.81)$ & $166.46(143.71,186.50)$ & 0.404 \\
Carbohydrate (g) & $59.16(52.39,74.36)$ & $61.02(52.46,74.32)$ & $61.02(46.04,68.38)$ & 0.307 \\
Protein(g) & $52.38(42.93,69.32)$ & $52.38(44.35,60.15)$ & $52.50(37.77,55.08)$ & 0.505 \\
Fat (g) & $6.09(4.89,9.58)$ & $6.09(5.4,9.15)$ & $6.42(4.92,8.90)$ & 0.365 \\
Fiber (g) & $44.18(40.16,93.09)$ & $44.18(48.38,91.34)$ & $54.90(37.5,69.26)$ & 0.396 \\
Sugar (g) & $16.10(12.18,20.56)$ & $16.10(12.35,19.27)$ & $15.93(12.4,15.61)$ & 0.330 \\
Saturated fat (g) & $1388.28(1182.42,1719.00)$ & $1427.81(1318.17,1585.92)$ & $1482.45(1089.36,1448.69)$ & 0.341 \\
Energy (kcal) & & &
\end{tabular}

3

4 All values are expressed as median and interquartile range (IQR) 


\section{Table 3(on next page)}

Short chain fatty acid concentration of feces at baseline, testing and washout.

*Significant difference between sample groups indicated by the Kruskal-Wallis test ( $p \leq$ 0.05). Descriptive statistic, interquartile range (IQR) 
1 Table 3 Short chain fatty acid concentration of feces at baseline, testing and washout.

\begin{tabular}{|c|c|c|c|c|c|c|c|c|}
\hline \multirow[b]{2}{*}{ SCFA } & \multirow[b]{2}{*}{ Time Point } & \multirow{2}{*}{$\begin{array}{c}\text { Placebo } \\
\text { Median (IQR) } \\
(\mu \mathrm{mol} / \mathrm{g} \text { feces })\end{array}$} & \multirow{2}{*}{$\begin{array}{c}\text { 3CMH } \\
\text { Median (IQR) } \\
\text { ( } \mu \mathrm{mol} / \mathrm{g} \text { feces) }\end{array}$} & \multirow{2}{*}{$\begin{array}{c}\text { 5CMH } \\
\text { Median (IQR) } \\
(\mu \mathrm{mol} / \mathrm{g} \text { feces }) \\
\end{array}$} & \multicolumn{4}{|c|}{$p$-value } \\
\hline & & & & & $\begin{array}{l}\text { P vs } 3 \mathrm{CMH} \\
\text { vs } 5 \mathrm{CMH}\end{array}$ & P vs 3СМH & P vs 5CMH & $\begin{array}{l}3 \mathrm{CMH} \text { vs } \\
\mathbf{5 C M H}\end{array}$ \\
\hline \multirow{3}{*}{ Lactic acid } & Baseline & $17.15(11.84,23.31)$ & $21.35(18.41,24.24)$ & $21.89(17.34,39.97)$ & 0.125 & 0.193 & 0.058 & 0.347 \\
\hline & Testing & $19.68(15.02,27.97)$ & $30.67(20.21,36.7)$ & $17.69(16.44,28.13)$ & 0.123 & 0.098 & 0.65 & 0.068 \\
\hline & Washout & $17.7(4.79,21)$ & $24.36(19.11,31.62)$ & $26.47(20.87,29.22)$ & $0.015^{*}$ & 0.057 & $0.005^{*}$ & 0.664 \\
\hline \multirow{3}{*}{ Acetic acid } & Baseline & $58.21(50.51,79.36)$ & $61.42(48.41,79.1)$ & $55.4(47.16,66.76)$ & 0.588 & 0.866 & 0.377 & 0.366 \\
\hline & Testing & $64.28(45.18,70.16)$ & $52.72(44.39,69.98)$ & $51.18(44.13,62.7)$ & 0.349 & 0.465 & 0.135 & 0.552 \\
\hline & Washout & $61.78(46.2,75.22)$ & $61.78(41.43,77.79)$ & $53.83(48.95,71.56)$ & 0.947 & 0.866 & 0.927 & 0.716 \\
\hline \multirow{3}{*}{ Propionic acid } & Baseline & $23.72(16.49,27.18)(n=19)$ & $23.12(15.86,28.83)$ & $24.15(18.71,34.85)$ & 0.604 & 0.942 & 0.311 & 0.476 \\
\hline & Testing & $25.63(17.58,30.68)$ & $25.16(17.11,29.15)$ & $23.33(15.82,26.5)$ & 0.493 & 0.8 & 0.273 & 0.35 \\
\hline & Washout & $24.35(17.16,28.04)$ & $24.86(17.95,31.01)$ & $24.74(21.76,28.49)$ & 0.725 & 0.536 & 0.465 & 0.862 \\
\hline \multirow{3}{*}{ Butyric acid } & Baseline & $29.2(20.1,37.1)$ & $29.36(24.26,41.67)$ & $29.42(18.08,36.87)$ & 0.992 & 0.955 & 0.879 & 1 \\
\hline & Testing & $32.48(24.5,39.48)$ & $27.55(22.02,41.36)$ & $32.94(24.71,45)$ & 0.656 & 0.339 & 0.784 & 0.623 \\
\hline & Washout & $35.69(25.48,40.45)$ & $26.24(21.54,32)$ & $28.34(23.84,38.21)$ & 0.2 & $0.049^{*}$ & 0.537 & 0.476 \\
\hline
\end{tabular}

3 *Significant difference between sample groups indicated by the Kruskal-Wallis test $(p \leq 0.05)$.

4 Descriptive statistic, interquartile range (IQR) 


\section{Table 4 (on next page)}

Quantity of Immunoglobulin A (IgA) in stool samples.

* indicates significant $(p \leq 0.05)$ difference. Descriptive statistic, interquartile range (IQR) 
1 Table 4 Quantity of Immunoglobulin A (IgA) in stool samples.

\begin{tabular}{|c|c|c|c|c|}
\hline \multirow[b]{2}{*}{ Stool IgA } & Placebo $(n=19)$ & 3 CMH $(n=19)$ & 5 CMH $(n=17)$ & \multirow{2}{*}{$p$-value } \\
\hline & Median (pg/mL) (IQR) & Median (pg/mL) (IQR) & Median (pg/mL) (IQR) & \\
\hline Baseline & $79069(13216,331439.01)$ & $22441.1(7789.4,332368.98)$ & $33852.5(13573.8,137549.8)$ & 0.654 \\
\hline Testing & $50919.7(13580.8,332101.51)$ & $37519.6(10274.7,331891.65)$ & $14500.4(3156.4,39546.6)$ & 0.147 \\
\hline Median change (IQR) & $-2914.7(-241278.22,3065.58)$ & $2322.3(-11346.5,11236.6)$ & $-10800.2(-88488.5,-1495.07)$ & 0.190 \\
\hline$p$-value ${ }^{\neq}$ & 0.198 & 0.687 & 0.013 & \multirow{3}{*}{ p-value } \\
\hline \multirow[b]{2}{*}{ Change of stool IgA } & Placebo $(n=19)$ & 3 CMH $(n=19)$ & $5 \mathrm{CMH}(\mathrm{n}=17)$ & \\
\hline & $\mathrm{N}(\%)$ & $\mathrm{N}(\%)$ & $\mathrm{N}(\%)$ & \\
\hline Increase & $4(21.1 \%)$ & $8(42.1 \%)$ & $3(17.6 \%)$ & 0.195 \\
\hline Decrease & $8(42.1 \%)$ & $5(26.3 \%)$ & $12(70.6 \%)$ & $0.027^{*}$ \\
\hline Same & $7(36.8 \%)$ & $6(31.6 \%)$ & $2(11.8 \%)$ & 0.211 \\
\hline
\end{tabular}

2

$3 *$ indicates significant $(p \leq 0.05)$ difference.

4 Descriptive statistic, interquartile range (IQR) 
Figure 1

Flow chart design of the copra meal hydrolysate $(\mathrm{CMH})$ study.

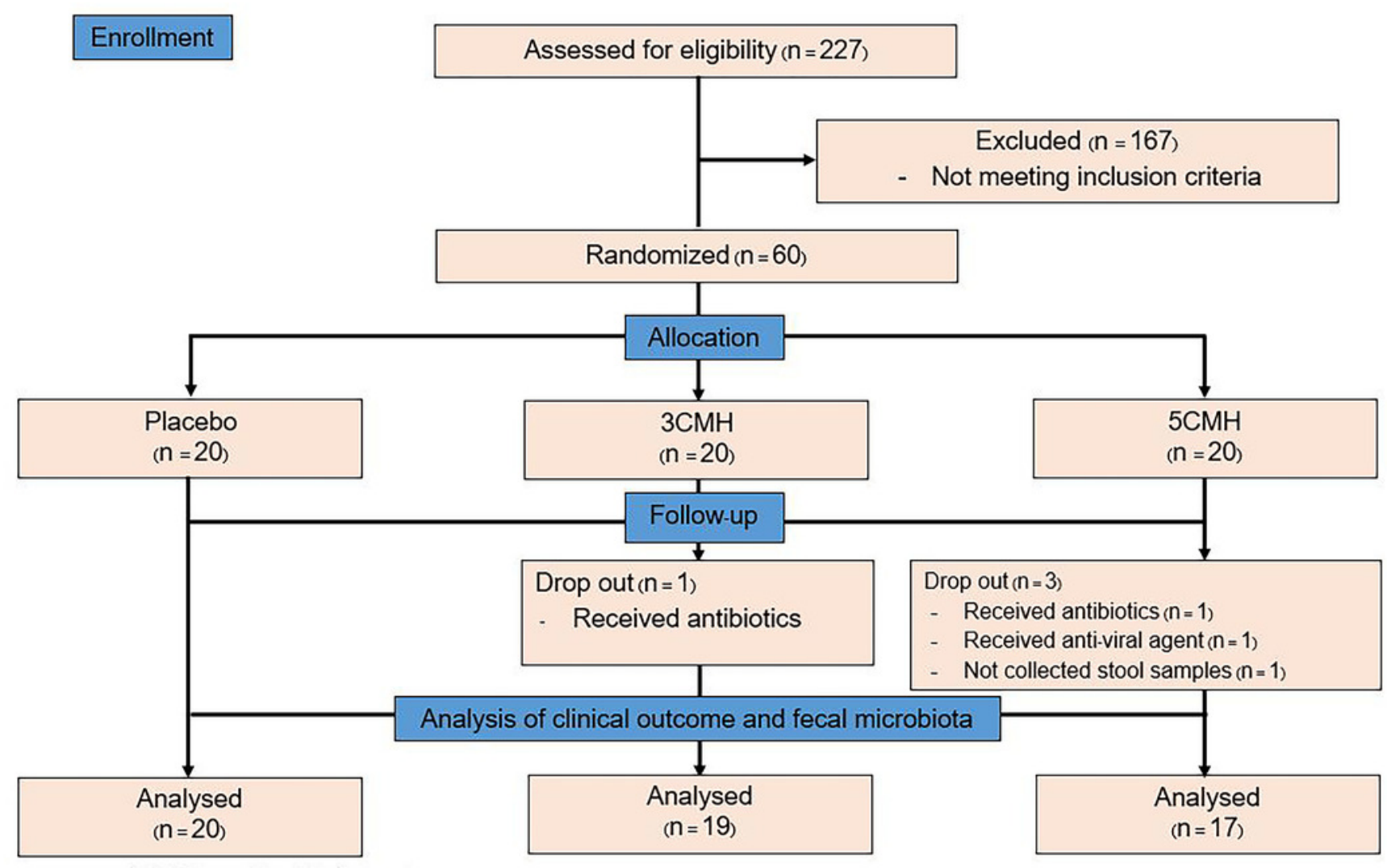


Figure 2

Timeline of copra meal hydrolysate $(\mathrm{CMH})$ studied, including sample groups and fecal collection.

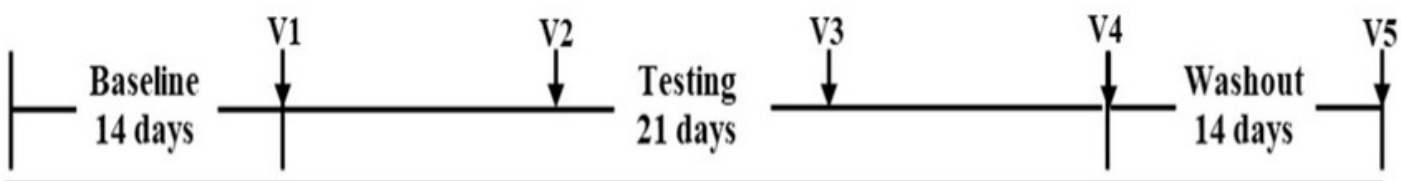

Placebo

Placebo (10 g maltodextrin)

$3 \mathrm{CMH}$

$3 \mathrm{~g}$ CHM and $7 \mathrm{~g}$ maltodextrin

$5 \mathrm{CMH}$

$5 \mathrm{~g} \mathrm{CHM}$ and 5 g maltodextrin

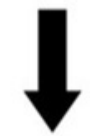

Fecalcollection

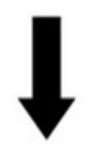

Fecal collection Fecal collection 
Figure 3

Significant change of defecating condition and gastrointestinal symptoms of placebo, $3 \mathrm{CMH}$ and $5 \mathrm{CMH}$ groups $(\mathrm{p} \leq 0.059)$.

Comparison between the three groups, different letters $(a, b)$ show significant difference $(p \leq$ $0.05)$.
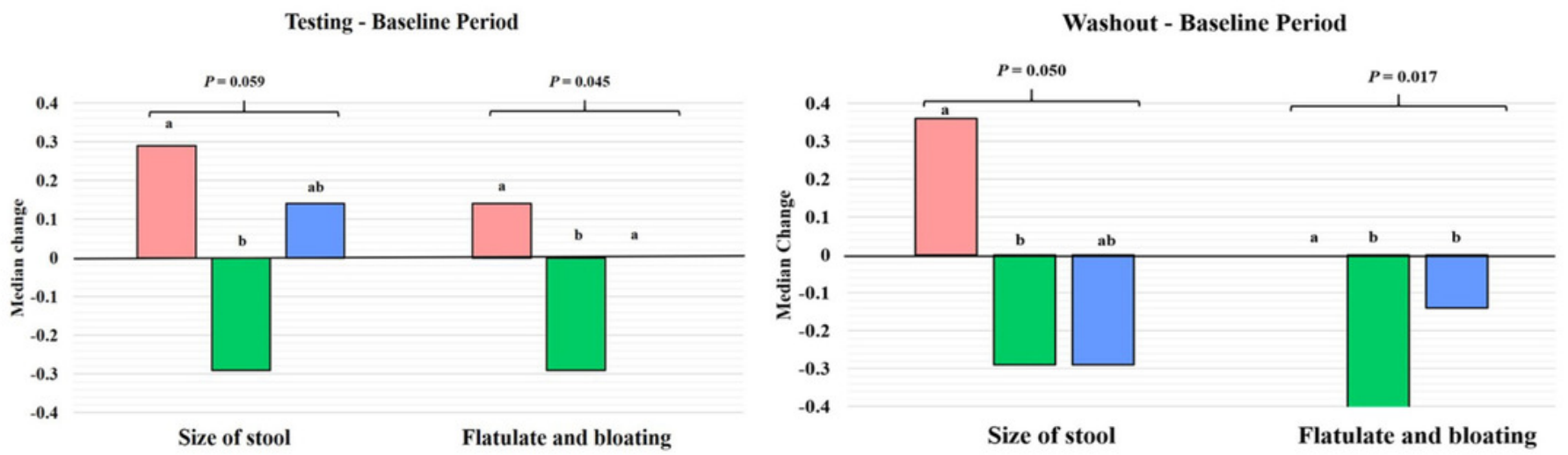

Placebo $\square$ 3CMH $\square$ 5CMH 
Figure 4

Change of gut microbiota at family level for testing $(A)$ and washout period $(B)$ of placebo, $3 \mathrm{CMH}$ and $5 \mathrm{CMH}$ groups compared to the baseline.

* Significant difference between placebo, $3 \mathrm{CMH}$ and $5 \mathrm{CMH}$ groups is shown by different letters $(p \leq 0.05)$.

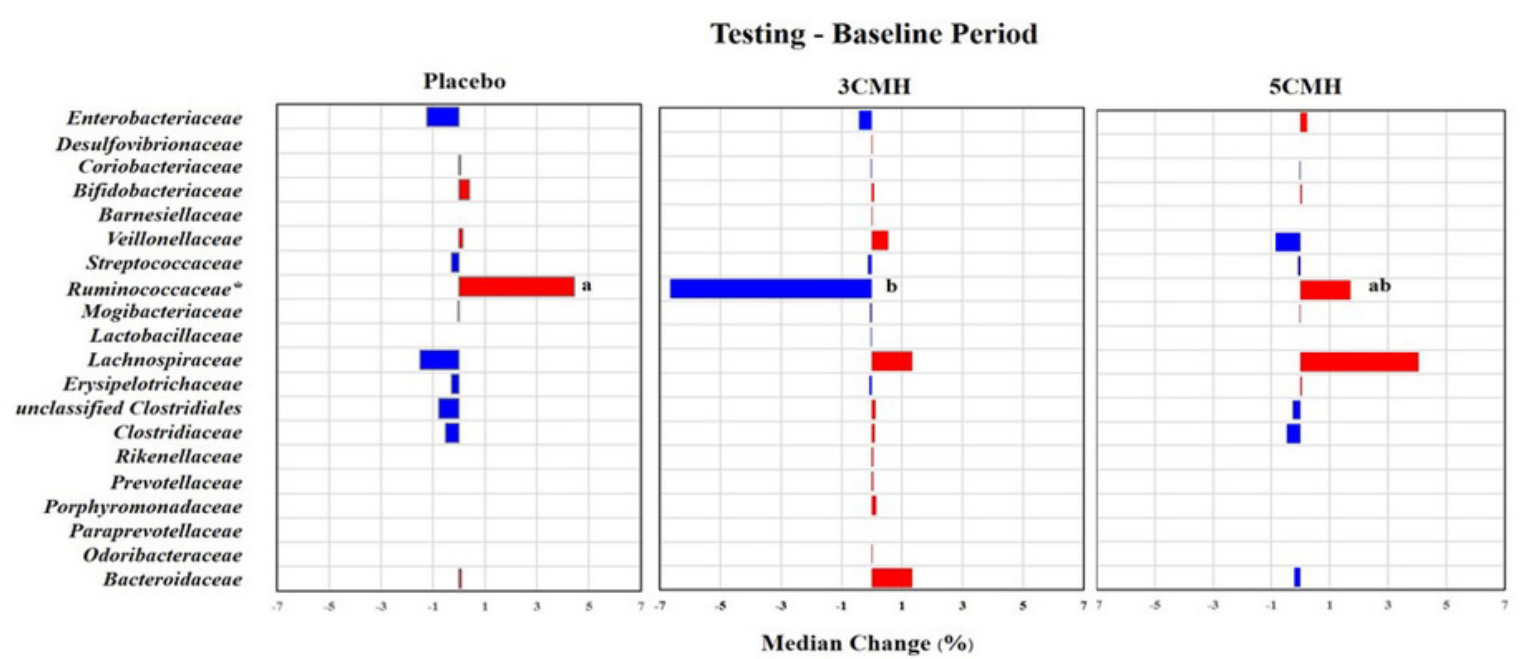

Decrease

Washout - Baseline Period

Increase

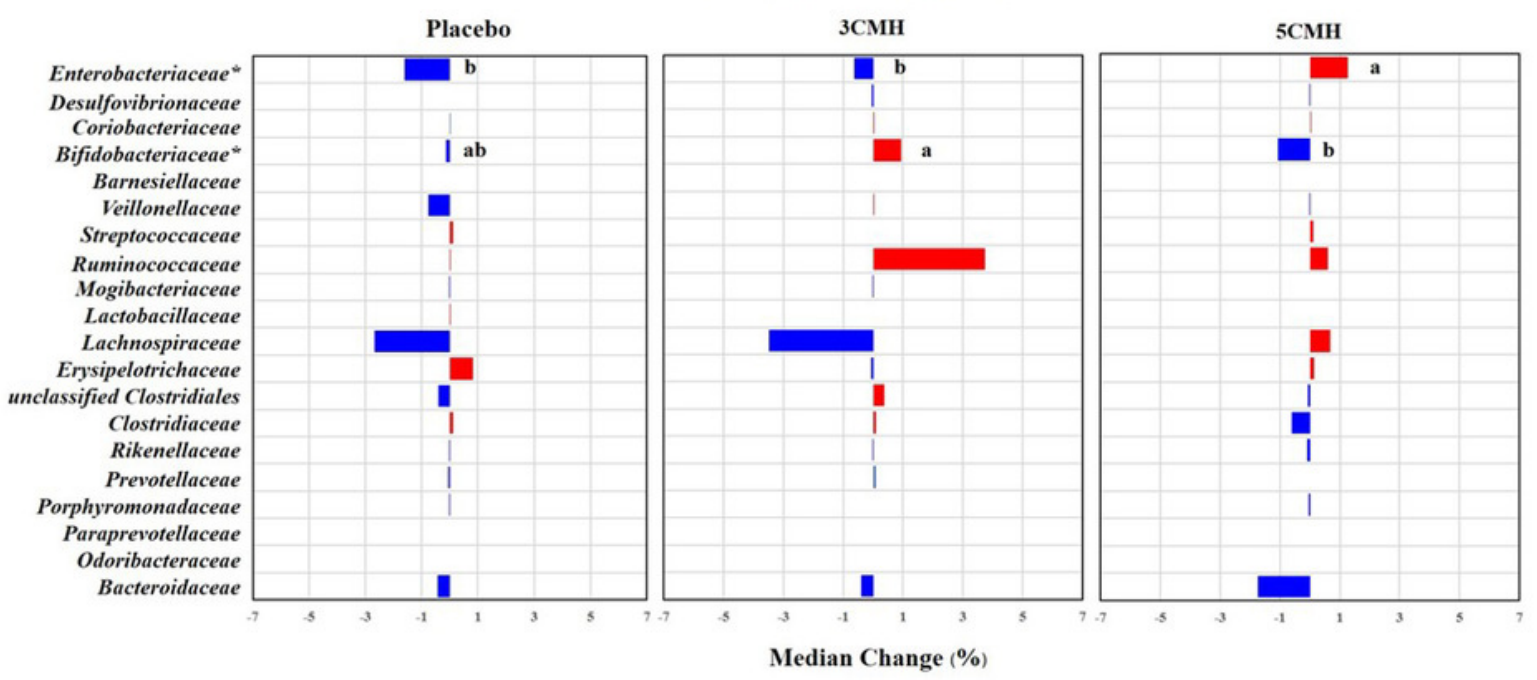


Figure 5

Prediction of change in carbohydrate related metabolism by PICRUSt analysis from baseline to testing period between placebo (white), 3CMH (gray) and 5CMH (black) groups.

* Significant difference between sample groups is shown by different letters ( $p \leq 0.05$ ).

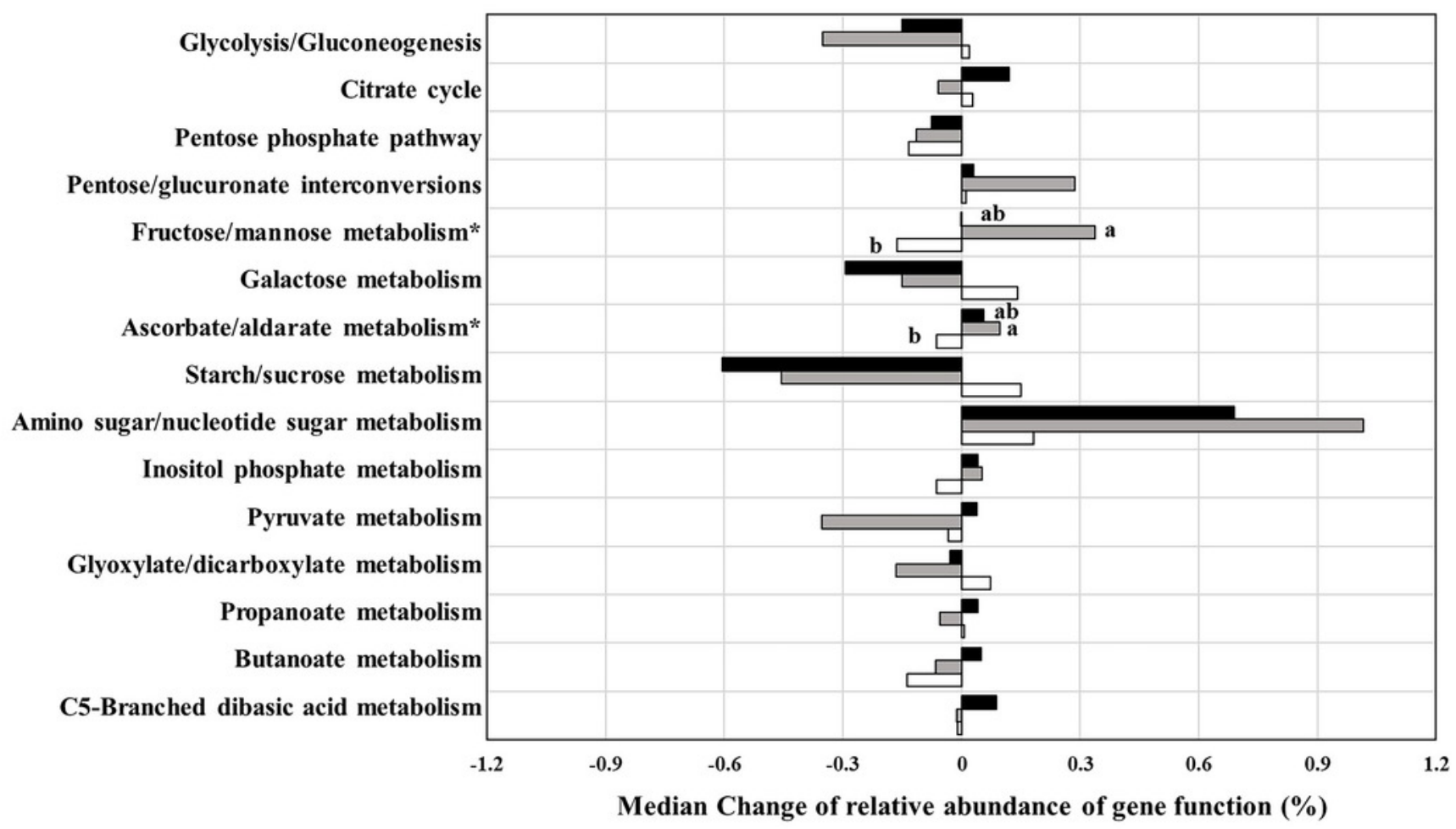

